\title{
On the period function in a class of generalized Lotka-Volterra systems
}

\author{
J. Villadelprat \\ Departament de Matemàtica Aplicada i Anàlisi, \\ Universitat de Barcelona, \\ Gran Via 585, 08007 Barcelona, Spain
}

\begin{abstract}
In this note, motivated by the recent results of Wang, Chen and Zhang in [Local bifurcations of critical periods in a generalized 2D LV system, Appl. Math. Comput. 214 (2009), 17-25], we study the behaviour of the period function of the center at the point $(1,1)$ of the planar differential system

$$
\left\{\begin{array}{l}
u^{\prime}=u^{p}\left(1-v^{q}\right), \\
v^{\prime}=\mu v^{q}\left(u^{p}-1\right),
\end{array}\right.
$$

where $p, q, \mu \in \mathbb{R}$ with $p q>0$ and $\mu>0$. Our aim is twofold. Firstly, we determine regions in the parameter space for which the corresponding system has a center with a monotonic period function. Secondly, by taking advantage of the results of Wang et al., we show some properties of the bifurcation diagram of the period function and we make some comments for further research. The differential system under consideration is a generalization proposed by Farkas and Noszticzius of the Lotka-Volterra model.
\end{abstract}

\section{Introduction and statement of the result}

Simple nonlinear dynamic models with periodic solutions have a great importance in describing complex dissipative systems, especially in chemistry and biology. The Lotka-Volterra model is a classical example and it is widely used to describe chemical and biological systems that oscillate.

The generalization of the Lotka-Volterra model proposed by Farkas and Noszticzius in [9] contains three chemical reactions: (GLV1) autocatalytic production of the intermediate $X$, (GLV2) autocatalytic transformation of $X$ into $Y$, and (GLV3) decay of the intermediate $Y$. It can be formulated by the following reaction schemes:

$$
\begin{aligned}
& A+p X \stackrel{k_{1}}{\longrightarrow}(p+1) X, \quad(\text { GLV1) } \\
& p X+q Y \stackrel{k_{2}}{\longrightarrow}(q+1) Y, \quad \text { (GLV2) } \\
& q Y \stackrel{k_{3}}{\longrightarrow} B, \quad \text { (GLV3) }
\end{aligned}
$$

where $X$ and $Y$ are the intermediates, $A$ and $B$ are reactants of constant concentration, $p$ and $q$ are respectively the molecules of $X$ and $Y$ that participate in one elementary reaction, and $k_{i}$ (for $i=1,2,3$ ) is the reaction rate of the $i$ th step. By simple law of mass action principles, the kinetics of these schemes can be described by the following system of ordinary differential equations:

$$
\left\{\begin{array}{l}
\dot{x}=k_{1} a x^{p}-p k_{2} x^{p} y^{q} \\
\dot{y}=k_{2} x^{p} y^{q}-q k_{3} y^{q}
\end{array}\right.
$$

2000 AMS Subject Classification: 34C25; 34C07; 92E20; 80A30.

Key words and phrases: Lotka-Volterra model, center, period function, critical period.

The author is partially supported by the CONACIT through the grant 2005-SGR-00550 and by the DGES through the grant MTM-2005-06098-C02-1. 
where $x$ and $y$ are respectively the concentrations of the intermediates $X$ and $Y$, the dot denotes derivation with respect to time $t$, and $a$ is the concentration of $A$. The authors in [7] show that the transformation

$$
\left\{u=\left(\frac{k_{2}}{q k_{3}}\right)^{1 / p} x, v=\left(\frac{p k_{2}}{a k_{1}}\right)^{1 / q} y, s=a k_{1}\left(\frac{q k_{3}}{k_{2}}\right)^{1-1 / p} t\right\}
$$

brings system (1) to

$$
\left\{\begin{array}{l}
u^{\prime}=u^{p}\left(1-v^{q}\right) \\
v^{\prime}=\mu v^{q}\left(u^{p}-1\right),
\end{array}\right.
$$

where $\mu:=\frac{1}{p}\left(\frac{p k_{2}}{a k_{1}}\right)^{1 / q}\left(\frac{q k_{3}}{k_{2}}\right)^{1 / p}$ and the prime denotes derivation with respect to $s$. If all the reactions are of the first order with respect to any intermediate, then system (2) reduces to the classical Lotka-Volterra system $(p=q=1)$. The generalized system (2) includes other important chemical models as special cases (see $[8,9,10,26])$.

In this note we shall assume that $p, q \in \mathbb{R}$ with $p q>0$, although, from the point of view of the chemical model, $p$ and $q$ should be natural numbers. We shall suppose in addition that $\mu$ is positive and independent of $p$ and $q$. (This setting is slightly more general than the original one but it is also interesting from the theoretical point of view.) Under these assumptions the differential system is analytic on the (open) first quadrant no matter that $p$ or $q$ are negative, and the critical point at $(u, v)=(1,1)$ is a center. Recall that a critical point $z$ of a planar differential system is a center if it has a neighbourhood $U$ such that $U \backslash\{z\}$ consists entirely of periodic orbits surrounding $z$. The largest punctured neighbourhood with this property is called the period annulus of the center and, in what follows, it will be denoted by $\mathcal{P}$. The period function of the center assigns to each periodic orbit in $\mathcal{P}$ its period. Questions related to the behaviour of the period function have been extensively studied. Let us quote, for instance, the problems of isochronicity (see $[4,6,14]$ ), monotonicity (see [2, 22, 28]) or bifurcation of critical periods (see [1, 3, 12, 13, 19, 27, 30]).

The period function of a center is monotonous increasing (respectively, decreasing) if for any pair of periodic orbits inside $\mathcal{P}$, say $\gamma_{1}$ and $\gamma_{2}$ with $\gamma_{1} \subset \operatorname{Int}\left(\gamma_{2}\right)$, we have that the period of $\gamma_{2}$ is greater (respectively, smaller) than the one of $\gamma_{1}$. (Here by $\operatorname{Int}(\gamma)$ we mean the bounded connected component of $\mathbb{R}^{2} \backslash\{\gamma\}$.) If all the periodic orbits in $\mathcal{P}$ have the same period, then the center is said to be isochronous.

The following is our main result and in its statement $\mathscr{C}$ and $\mathscr{D}$ stand respectively for the light grey and dark grey regions in Figure 1. To be more precise, denoting $I^{2}=I \times I$,

$$
\mathscr{C}=[-1,0)^{2} \cup[1 / 2,+\infty)^{2} \backslash\left\{i_{1}, i_{3}\right\} \text { and } \mathscr{D}=(-\infty,-1]^{2} \cup(0,1 / 2]^{2} \backslash\left\{i_{1}, i_{3}\right\} .
$$

With this notation, the aim of the present note is to prove the following:

Theorem A. Let us take $p, q, \mu \in \mathbb{R}$ with $p q>0$ and $\mu>0$ and consider the planar differential system (2). Then, if $(p, q)$ belongs to $\mathscr{C}$ (respectively, $\mathscr{D}$ ), the period function of the center at $(1,1)$ is monotonous increasing (respectively, decreasing). In addition, if $(p, q) \in\left\{i_{1}, i_{3}\right\}$, then the center is isochronous.

In fact, taking the period constants into account (see Section 3), it turns out that $i_{1}$ and $i_{3}$ are the only isochronous centers of system (2). On the contrary, numerical evidences show that there are parameters not in $\mathscr{C} \cup \mathscr{D}$ for which the associated center has a monotonic period function (see also Section 3).

There are several previous papers to be quoted in relation with the results in Theorem A. As we have already mentioned, the case $p=q=1$ corresponds to the original Lotka-Volterra system and the fact that its center has a monotonous increasing period function was proved independently by Rothe [18], Schaaf [20] and Waldvogel [24] in mid-eighties. More recently, the bifurcation of critical periods of system (2) at the inner boundary of the period annulus (i.e., the center itself) has been studied in [25]. In short (see Section 3 for details), the authors prove that at most two critical periods bifurcate from a weak center of finite order. In case of weak centers of infinite order (i.e., the isochrones), this upper bound is one for $\mu \neq 1$ and two for $\mu=1$. This implies, for instance, that near the isochrones $i_{1}$ and $i_{3}$ (see Figure 1) there are parameters 


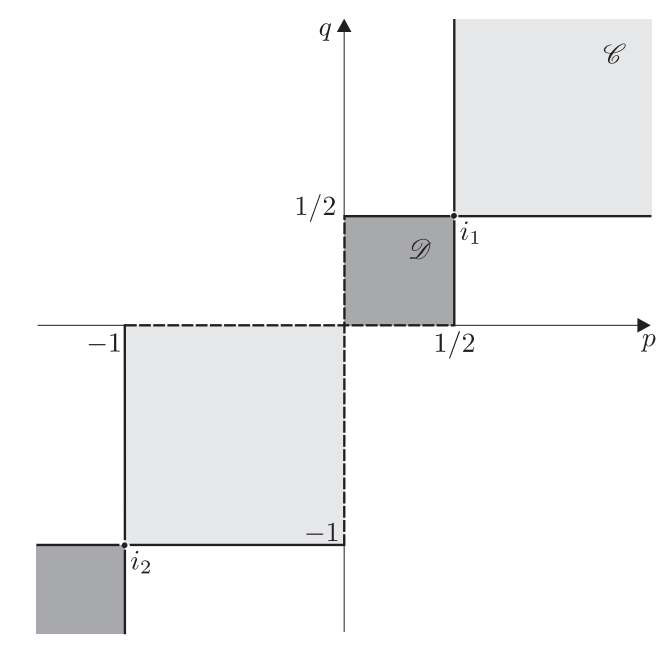

Figure 1: Monotonicity regions in Theorem A.

$(p, q)$ for which the corresponding center has a non-monotonic period function. (Of course, according to Theorem A, these parameters are outside $\mathscr{C} \cup \mathscr{D}$.) We stress that the approach in [25] is different from the one here because in that paper the authors study the local behaviour of the period function near the center. The monotonicity in Theorem A refers to its behaviour in the whole period annulus.

Let us conclude this introduction by mentioning that

$$
\left\{\begin{array}{l}
\dot{x}=-y-b x^{2}-c x y+b y^{2} \\
\dot{y}=x(1+y)
\end{array}\right.
$$

is also referred as "generalized Lotka-Volterra center" in several papers dealing with the so-called Hilbert's sixteenth problem (see for instance [29] and papers quoting it). This is so because this 2-parametric family also includes the classical Lotka-Volterra center, which corresponds to $b=0$. In this setting it is to be quoted the results in [23], where it is proved that the center of the above system has a monotonic period function as well for those parameters such that $b=-1$ or $c^{2}+(2 b+1)^{2}=1$.

The paper is organized as follows. Section 2 is devoted to the proof of the result. In Section 3 we make some remarks for further research in the regions not covered by Theorem A.

\section{Proof of the result}

The key point to prove Theorem A is that there exists a coordinate transformation that brings system (2) to a Hamiltonian system with separate variables. Next lemma explains how we found this change of variables.

Lemma 2.1. Assume that a given planar differential system in $U \subset \mathbb{R}^{2}$ has a first integral $\widehat{H}$ and let $\eta$ be its corresponding integrating factor. In other words, let us consider

$$
\left\{\begin{array}{l}
\dot{u}=-\widehat{H}_{v}(u, v) / \eta(u, v), \\
\dot{v}=\widehat{H}_{u}(u, v) / \eta(u, v)
\end{array}\right.
$$

Suppose that there exists a diffeomorphism $\psi$ in $U$ such that $\operatorname{det}(D \psi)_{(u, v)}=\eta(u, v)$ for all $(u, v) \in U$. Then the coordinate transformation $(x, y)=\psi(u, v)$ brings (3) to the Hamiltonian system

$$
\left\{\begin{array}{l}
\dot{x}=-H_{y}(x, y) \\
\dot{y}=H_{x}(x, y)
\end{array}\right.
$$


where $H=\widehat{H} \circ \psi^{-1}$.

Proof. In fact we will show that $(u, v)=\psi^{-1}(x, y)$ brings (4) to (3). To this end define $J=\left(\begin{array}{cc}0 & -1 \\ 1 & 0\end{array}\right)$, so that system (4) writes as $(\dot{x}, \dot{y})^{t}=J \nabla H^{t}(x, y)$. Then

$$
\begin{aligned}
\left(\begin{array}{c}
\dot{u} \\
\dot{v}
\end{array}\right) & =\left(D \psi^{-1}\right)_{(x, y)}\left(\begin{array}{c}
\dot{x} \\
\dot{y}
\end{array}\right)=\left(D \psi^{-1}\right)_{\psi(u, v)} J \nabla H^{t}(x, y)=(D \psi)_{(u, v)}^{-1} J \nabla H^{t}(x, y) \\
& \left.=\frac{1}{\operatorname{det}(D \psi)_{(u, v)}}\left(\begin{array}{rr}
\psi_{2_{v}} & -\psi_{2_{u}} \\
-\psi_{1_{v}} & \psi_{1_{u}}
\end{array}\right)_{(u, v)}^{t} J \nabla H^{t}(x, y)=\frac{1}{\eta(u, v)}\left(\nabla H(x, y) J^{t}\left(\begin{array}{rr}
\psi_{2_{v}} & -\psi_{2_{u}} \\
-\psi_{1 v} & \psi_{1_{u}}
\end{array}\right)_{(u, v)}\right)\right)^{t} .
\end{aligned}
$$

Now, since one can verify that

$$
J^{t}\left(\begin{array}{rr}
\psi_{2 v} & -\psi_{2 u} \\
-\psi_{1_{v}} & \psi_{1_{u}}
\end{array}\right)_{(u, v)}=-(D \psi)_{(u, v)} J
$$

from the above equality it follows that $(\dot{u}, \dot{v})=\frac{-1}{\eta(u, v)} \nabla H(x, y)(D \psi)_{(u, v)} J$. Hence, on account of

$$
\nabla H(x, y)=\nabla \widehat{H}\left(\psi^{-1}(x, y)\right)\left(D \psi^{-1}\right)_{(x, y)}=\nabla \widehat{H}(u, v)\left(D \psi^{-1}\right)_{\psi(u, v)}=\nabla \widehat{H}(u, v)(D \psi)_{(u, v)}^{-1},
$$

it turns out that $(\dot{u}, \dot{v})=\frac{-1}{\eta(u, v)} \nabla \widehat{H}(u, v) J=\frac{1}{\eta(u, v)} \nabla \widehat{H}(u, v) J^{t}$. This is precisely the transpose of (3).

Next, thanks to the previous result, we will obtain the above mentioned change of variables. This is done in the following lemma. Although it is a trivial result, its statement is a little cumbersome because we want to avoid treating the different cases separately. To this end we introduce this definition:

Definition 2.2 From now on we shall use the following notation:

$$
\mathscr{R}(s ; \kappa):= \begin{cases}(1+\kappa s)^{1 / \kappa}-s-1 & \text { if } \kappa \neq 0, \\ e^{s}-s-1 & \text { if } \kappa=0 .\end{cases}
$$

Sometimes, for the sake of shortness, we shall write $\mathscr{R}_{\kappa}(s)=\mathscr{R}(s ; \kappa)$.

Lemma 2.3. Define $\psi(u, v)=(h(u ; 1-p), h(v ; 1-q))$ where

$$
h(s ; \kappa)= \begin{cases}\frac{s^{\kappa}-1}{\kappa} & \text { if } \kappa \neq 0, \\ \log s & \text { if } \kappa=0 .\end{cases}
$$

Then the coordinate transformation $(x, y)=\psi(u, v)$ brings system (2) to

$$
\left\{\begin{array}{l}
\dot{x}=-G^{\prime}(y) \\
\dot{y}=F^{\prime}(x)
\end{array}\right.
$$

with $F(x)=\mu \mathscr{R}(x ; 1-p)$ and $G(y)=\mathscr{R}(y ; 1-q)$.

Proof. Let us suppose first that $p \neq 1$ and $q \neq 1$. Note that $\eta(u, v)=\frac{1}{u^{p} v^{q}}$ is an integrating factor of system (2) and that its corresponding first integral is given by

$$
\widehat{H}(u, v)=\mu\left(u-\frac{u^{1-p}-1}{1-p}-1\right)+v-\frac{v^{1-q}-1}{1-q}-1 .
$$


(Let us point out that here we took the first integral vanishing at the point $(1,1)$ for the sake of convenience.) Clearly, $\psi(u, v)=\left(\frac{u^{1-p}-1}{1-p}, \frac{v^{1-q}-1}{1-q}\right)$ is a diffeomorphism verifying $\operatorname{det}(D \psi)_{(u, v)}=\eta(u, v)$ for all $(u, v)$. Thus, by applying Lemma 2.1 , the coordinate transformation $(x, y)=\psi(u, v)$ brings system (2) to the Hamiltonian system associated to

$$
H(x, y)=\widehat{H}\left(\psi^{-1}(x, y)\right)=\mu\left((1+(1-p) x)^{\frac{1}{1-p}}-x-1\right)+(1+(1-q) y)^{\frac{1}{1-q}}-y-1 .
$$

This, according to Definition 2.2, proves the result when $(p-1)(q-1) \neq 0$.

The case $(p-1)(q-1)=0$ splits up in three subcases, namely: $\{p=1, q \neq 1\},\{p \neq 1, q=1\}$ and $\{p=1, q=1\}$. The coordinate transformation and the Hamiltonian function in each subcase can be obtained by taking the limits $p \longrightarrow 1$ or $q \longrightarrow 1$ in the functions of the case $(p-1)(q-1) \neq 0$. The verification of the result in these subcases is straightforward and it is left to the reader.

Next we shall apply a monotonicity criterion for the period function of Hamiltonian systems with separate variables. In order to state it, the following definition is needed:

Definition 2.4 Let $\Omega$ be an open interval of $\mathbb{R}$ and let us consider a smooth function $f: \Omega \longrightarrow \mathbb{R}$. Define $\Psi(f)=\left(f /\left(f^{\prime}\right)^{2}\right)^{\prime \prime}$. We say that $f \in \mathcal{I}(\Omega)$ (respectively, $f \in \mathcal{D}(\Omega)$ ) if $\Psi(f)$ is positive (respectively, negative) on $\Omega$. Moreover, we say that $f \in \mathcal{N}(\Omega)$ if $\Psi(f) \equiv 0$ on $\Omega$.

Let $F$ and $G$ be smooth functions with a non-degenerated local minimum at 0 . Then the Hamiltonian differential system

$$
\left\{\begin{array}{l}
\dot{x}=-G^{\prime}(y), \\
\dot{y}=F^{\prime}(x),
\end{array}\right.
$$

has a center at the origin. Let $P_{x}$ and $P_{y}$ be the projections of its period annulus on the $x$-axis and $y$-axis respectively. The proof of the following result can be found in $[5,11]$.

Proposition 2.5. Consider the center at the origin of the Hamiltonian differential system (5).

(a) If $(F, G) \in \mathcal{N}\left(P_{x}\right) \times \mathcal{N}\left(P_{y}\right)$, then the center is isochronous.

(b) If $(F, G)$ belongs to $\mathcal{D}\left(P_{x}\right) \times \mathcal{D}\left(P_{y}\right), \mathcal{N}\left(P_{x}\right) \times \mathcal{D}\left(P_{y}\right)$ or $\mathcal{D}\left(P_{x}\right) \times \mathcal{N}\left(P_{y}\right)$, then the center has a monotonous decreasing period function.

(c) If $(F, G)$ belongs to $\mathcal{I}\left(P_{x}\right) \times \mathcal{I}\left(P_{y}\right), \mathcal{N}\left(P_{x}\right) \times \mathcal{I}\left(P_{y}\right)$ or $\mathcal{I}\left(P_{x}\right) \times \mathcal{N}\left(P_{y}\right)$, then the center has a monotonous increasing period function.

Next we study for which values of the parameter $\kappa$, if any, $\mathscr{R}_{\kappa}$ belongs to $\mathcal{D}$ or $\mathcal{I}$. This is done in the following lemma, where we set $\Omega_{\kappa}=(-1 / \kappa,+\infty)$ for $\kappa>0, \Omega_{\kappa}=(-\infty,-1 / \kappa)$ for $\kappa<0$ and $\Omega_{0}=\mathbb{R}$ (i.e., the domain of $\mathscr{R}_{\kappa}$ when $1 / \kappa \notin \mathbb{N}$ ).

Lemma 2.6. Consider the function $s \longmapsto \mathscr{R}_{\kappa}(s)$ introduced in Definition 2.2.

(a) If $\kappa \in\{1 / 2,1,2\}$, then $\mathscr{R}_{\kappa} \in \mathcal{N}\left(\Omega_{\kappa}\right)$.

(b) If $\kappa \in(-\infty, 1 / 2) \cup(1,2)$, then $\mathscr{R}_{\kappa} \in \mathcal{I}\left(\Omega_{\kappa}\right)$.

(c) If $\kappa \in(1 / 2,1) \cup(2,+\infty)$, then $\mathscr{R}_{\kappa} \in \mathcal{D}\left(\Omega_{\kappa}\right)$.

Proof. Let us assume $\kappa \neq 0$ first. Some computations show that

$$
\Psi\left(\mathscr{R}_{\kappa}\right)(x)=(\kappa-1) \frac{(1+\kappa x)^{1 / \kappa-4} \mathscr{A}(x)}{\left((1+\kappa x)^{1 / \kappa-1}-1\right)^{4}},
$$


where

$$
\mathscr{A}(x):=(2 \kappa-1)(1+\kappa x)^{2 / \kappa}+2(\kappa-2)((2 \kappa-1) x+1)(1+\kappa x)^{1 / \kappa}-(1+\kappa x)((\kappa-2) x+4 \kappa-5) .
$$

The above expression shows that $\Psi\left(\mathscr{R}_{\kappa}\right)=0$ for $\kappa \in\{1 / 2,1,2\}$ and this proves $(a)$. Hence from now on we assume that $\kappa \notin\{1 / 2,1,2\}$. By computing the Taylor series of $\mathscr{A}$ at $x=0$ we know that

$$
\mathscr{A}(x)=(\kappa-1)^{2}(2 \kappa-1)(2-\kappa) x^{4}+\mathrm{o}\left(x^{4}\right)
$$

and, on the other hand, one can verify that its third derivative is given by

$$
\mathscr{A}^{(3)}(x)=2(1-2 \kappa)(\kappa-2)(\kappa-1)(1+\kappa x)^{1 / \kappa-3} \underbrace{\left((\kappa+1) x+2-2(1+\kappa x)^{1 / \kappa}\right)}_{b(x)} .
$$

Note that $b(0)=0$ for all $\kappa$. We claim that $b$ has an additional zero in $\Omega_{\kappa}$ (which is negative) if, and only if, $\kappa \geqslant-1$. Since $b^{\prime}(x)=\kappa+1-2(1+\kappa x)^{1 / \kappa-1}$, it is clear that $b^{\prime}(x)=0$ if, and only if, $(1+\kappa x)^{1 / \kappa-1}=\frac{\kappa+1}{2}$. An easy study of this equation in terms of the parameter $\kappa$ shows that the derivative of $b$ does not vanish for $\kappa<-1$ and vanishes exactly once, at some negative value, for $\kappa \geqslant-1$. On account of this, and the behaviour of $b$ at the endpoints of $\Omega_{\kappa}$, the claim follows by applying Rolle's Theorem.

Taking (8) and the above claim into account, by applying Rolle's Theorem once again we can assert that $\mathscr{A}$ has at most five zeros. In view of $(7)$, four of them correspond to the zero of multiplicity four at $x=0$. The fifth zero may only exist in case that $\kappa \geqslant-1$ and it should be negative. A straightforward study of the behaviour of $\mathscr{A}$ at the endpoints of $\Omega_{\kappa}$ shows that in fact this fifth zero does not exist for any value of $\kappa$. In short, $\mathscr{A}$ only vanishes at the origin and it does it with multiplicity four. Consequently, from (6), $\Psi\left(\mathscr{R}_{k}\right)$ does not vanish. Finally, since one can easily check that $\Psi\left(\mathscr{R}_{k}\right)(0)=\frac{(\kappa-2)(2 \kappa-1)}{12(1-\kappa)}$, the statements in (b) and (c) follow.

Finally, if $\kappa=0$, then

$$
\Psi\left(\mathscr{R}_{0}\right)(x)=\frac{e^{2 x}-4(x-1) e^{x}-2 x-5}{e^{-x}\left(e^{x}-1\right)^{4}},
$$

which it is easy to show that it is positive for all $x \in \mathbb{R}$. This concludes the proof of the result.

Proof of Theorem A. The period annulus of the center at $(1,1)$ of the differential system (2) is inside the first quadrant. By applying Lemma 2.3, the coordinate transformation $(x, y)=\psi(u, v)$ brings $(2)$ to the Hamiltonian differential system

$$
\left\{\begin{array}{l}
\dot{x}=-G^{\prime}(y) \\
\dot{y}=F^{\prime}(x)
\end{array}\right.
$$

where $F(x)=\mu \mathscr{R}(x ; 1-p)$ and $G(y)=\mathscr{R}(y ; 1-q)$. Note that $\psi$ maps the center at $(1,1)$ to the origin, and that the first quadrant is mapped to the rectangle $\Omega_{1-p} \times \Omega_{1-q}$. (Here we use again the notation $\Omega_{\kappa}=(-1 / \kappa,+\infty)$ for $\kappa>0, \Omega_{\kappa}=(-\infty,-1 / \kappa)$ for $\kappa<0$ and $\Omega_{0}=\mathbb{R}$.) Therefore the period annulus $\mathcal{P}$ of the center at the origin of system (9) is inside the rectangle $\Omega_{1-p} \times \Omega_{1-q}$. In particular, its projection on the $x$-axis (respectively, $y$-axis) is inside $\Omega_{1-p}$ (respectively, $\Omega_{1-q}$ ).

Clearly, to prove the result we can consider system (9) instead of the original one because both systems are conjugated. The advantage of doing this is that then we can apply Proposition 2.5.

Since $\mu>0$, from Lemma 2.6 it follows that $F \in \mathcal{N}\left(\Omega_{1-p}\right)$ for $p \in\{-1,0,1 / 2\}, F \in \mathcal{I}\left(\Omega_{1-p}\right)$ for $p \in(-1,0) \cup(1 / 2,+\infty)$ and $F \in \mathcal{D}\left(\Omega_{1-p}\right)$ for $p \in(-\infty,-1) \cup(0,1 / 2)$. Obviously, replacing $p$ by $q$, exactly the same result holds for $G$. Accordingly, by applying Proposition 2.5 we can assert that the center at the point $(1,1)$ of system (2) is isochronous when $p=q=-1$ or $p=q=1 / 2$, and that its period function is monotonous increasing for $(p, q) \in \mathscr{C}$ and monotonous decreasing for $(p, q) \in \mathscr{D}$. (The case $p q=0$ is not considered because then the critical point is not a center.) 


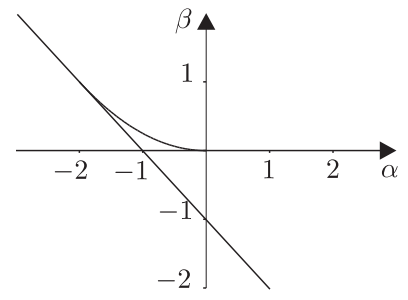

Figure 2: Bifurcation curves for the simplified model.

\section{Remarks for further research}

In the present paper we determine some regions in the parameter space

$$
\Pi:=\left\{\lambda=(p, q, \mu) \in \mathbb{R}^{3}: \mu>0, p q>0\right\}
$$

where the associated differential system (2) has a monotonic period function. Our aim in this final section is to show that the situation is not so simple for the parameters not covered by Theorem A, and to make some remarks for further research. More concretely, the problem that we pose is to obtain the bifurcation diagram of the period function of family of differential systems (2). In other words, to split the parameter space up as $\Pi=\bigcup V_{i}$ in such a way that if $\lambda_{1}$ and $\lambda_{2}$ belong to the same set $V_{i}$, then the associated period functions are qualitatively the same. In order to be more precise it is first necessary to parametrize the set of periodic orbits. To this end recall that the period annulus $\mathcal{P}$ of the center at $(1,1)$ of the differential system (2) is inside the first quadrant. Thus, if $\left(u_{\ell}, u_{r}\right)$ is the projection of $\mathcal{P}$ on the $u$-axis, then we have that $0 \leqslant u_{\ell}<1<u_{r} \leqslant+\infty$. For each $s \in\left(0, u_{r}-1\right)$ and $\lambda \in \Pi$, let $P(s ; \lambda)$ be the period of the periodic orbit of $(2)$ passing through the point $(1+s, 1) \in \mathcal{P}$. This provides us with a parametrization of the period function for which $s \approx 0$ corresponds to periodic orbits near the center. The critical periods are the critical points of $s \longmapsto P(s ; \lambda)$, and it can be shown that their number, character (maximum or minimum) and distribution does not depend on the particular parametrization of the set of periodic orbits used. It is clear moreover that the study of the critical periods is essential to understand the qualitative properties of the period function.

The boundary of the period annulus $\mathcal{P}$ has two connected components; the center itself and a polycycle. We call them, respectively, the inner and outer boundary of the period annulus. As it is established in [16], the bifurcation diagram of the period function consists in three parts:

(a) Bifurcations of the period function at the inner boundary (i.e., the center).

(b) Bifurcations of the period function at the outer boundary (i.e., the polycycle)

(c) Bifurcations of the period function in the interior of the period annulus.

Roughly speaking the bifurcations at the inner (respectively, outer) boundary correspond to critical periods that emerge, or disappear, from the center (respectively, the polycycle). According to the above parametrization, to determine them it is necessary to study $P(s ; \lambda)$ for $s \approx 0$ and $s \approx u_{r}-1$. The bifurcations in the interior of the period annulus correspond to parameters for which two critical periods collapse disappearing in $\mathcal{P}$. (As a matter of fact, talking about the interior of $\mathcal{P}$ is an abuse of language because the period annulus is open by definition.)

To clarify these notions we can consider the bifurcation of zeros of the equation $x^{2}+\alpha x+\beta=0$ for $x \in(0,1)$ as a simplified model. In this model $\{\beta=0\},\{1+\alpha+\beta=0\}$ and $\left\{\alpha^{2}=4 \beta,-2<\alpha<0\right\}$ would correspond to the bifurcation curves in $(a),(b)$ and $(c)$ respectively (see Figure 2). 


\subsection{Bifurcations at the inner boundary}

It is well known that the function $s \longmapsto P(s ; \lambda)$ is analytic on the interval $\left(0, u_{r}-1\right)$. The key point to study the bifurcations at the inner boundary is that it can be extended analytically to $s=0$. We can thus consider its Taylor development at $s=0$,

$$
P(s ; \lambda)=\sum_{i \geqslant 0} \Delta_{i}(\lambda) s^{i}
$$

The coefficients $\Delta_{i}$ for $i \geqslant 1$ are called the period constants of the center, and it can be shown that they are polynomials in $\lambda$. By the Hilbert basis theorem there exists a natural number $N$ such that the ideal of all the period constants is finitely generated by the first $N$. Moreover, it turns out that $\Delta_{2 k+1} \in\left(\Delta_{2}, \Delta_{4}, \ldots, \Delta_{2 k}\right)$, the ideal generated by $\Delta_{2 i}$ for $i=1,2, \ldots, k$, and that the the first $k \geqslant 1$ such that $\Delta_{k} \neq 0$ is even. The center corresponding to the parameter value $\lambda_{\star}$ is said to be a weak center of order $k$ if

$$
\Delta_{1}\left(\lambda_{\star}\right)=\Delta_{2}\left(\lambda_{\star}\right)=\ldots=\Delta_{2 k+1}\left(\lambda_{\star}\right)=0 \text { and } \Delta_{2 k+2}\left(\lambda_{\star}\right) \neq 0 .
$$

In case that $\Delta_{k}\left(\lambda_{\star}\right)=0$ for all $k \geqslant 1$, we say that it is a weak center of infinite order, which of course correspond to the isochrones.

We can now summarize the results in [25] in the following statement, where local critical period refers to a critical period bifurcating at the inner boundary of the period annulus (see $[3,16]$ for a precise definition).

Theorem 3.1. Consider the period function of the differential system (2). Then the following hold:

(a) For $k=1,2$, at most $k$ local critical periods bifurcate from weak centers of finite order $k$. Moreover, for any $0 \leqslant n \leqslant k$, there are perturbations with exactly $n$ local critical periods.

(b) $\Delta_{k} \in\left(\Delta_{2}, \Delta_{4}, \Delta_{6}\right)$ for all $k \geqslant 1$. In particular, there are no weak centers of finite order $k \geqslant 3$.

(c) If $\mu=1$, then at most 2 local critical periods bifurcate from weak centers of infinite order. Moreover, for any $n=1,2$, there are perturbations with exactly $n$ local critical periods.

If $\mu \neq 1$, then at most 1 local critical period bifurcate from weak centers of infinite order. Moreover, there are perturbations with exactly 1 critical period.

In the following discussion we have used the explicit expression of $\Delta_{2}, \Delta_{4}$ and $\Delta_{6}$. We do not include them here for the sake of shortness (the interested reader can find them in [25]). For simplicity in the exposition we shall assume that the parameter $\mu>0$ is fixed. Thus, making an abuse of notation, we will write $\lambda=(p, q)$. Taking this into account, it is easy to show that

$$
\Lambda_{\infty}:=\left\{\lambda=(p, q) \in \mathbb{R}^{2}: \Delta_{2}(\lambda)=\Delta_{4}(\lambda)=\Delta_{6}(\lambda)=0\right\}=\left\{i_{1}, i_{2}, i_{3}, i_{4}\right\}
$$

where $i_{1}=\left(\frac{1}{2}, \frac{1}{2}\right), i_{2}=\left(-1, \frac{1}{2}\right), i_{3}=(-1,-1)$ and $i_{4}=\left(\frac{1}{2},-1\right)$. (Note that only $i_{1}$ and $i_{3}$ are "true" isochrones since $p q>0$ is a necessary condition in order to have a center.) Moreover, if $\mu \neq 1$, then

$$
\Lambda_{2}:=\left\{\lambda=(p, q) \in \mathbb{R}^{2}: \Delta_{2}(\lambda)=\Delta_{4}(\lambda)=0 \text { and } \Delta_{6}(\lambda) \neq 0\right\}=\left\{m_{1}, m_{2}, m_{3}\right\}
$$

It can be proved, see Figure 3 , that $m_{1}=m_{1}(\mu)$ is inside the square $(0,1)^{2}$ and that it tends to $i_{1}$ as $\mu \longrightarrow 1$. Similarly, $m_{3}=m_{3}(\mu)$ is inside the square $(-2,0)^{2}$ and it tends to $i_{3}$ as $\mu \longrightarrow 1$. Finally, $m_{2}=m_{2}(\mu)$ is in the second quadrant for $\mu \in(0,1)$ and in the forth quadrant for $\mu>1$. In addition, $\left\|m_{2}\right\| \longrightarrow+\infty$ as $\mu$ tends to 1 . In particular, it turns out that $\Lambda_{2}$ is empty for $\mu=1$. Setting

$$
\Lambda_{1}:=\left\{\lambda=(p, q) \in \mathbb{R}^{2}: \Delta_{2}(\lambda)=0 \text { and } \Delta_{4}(\lambda) \neq 0\right\},
$$

we have that $\Lambda_{k}$ is the set of weak centers of order $k$ for $k=1,2, \infty$. 


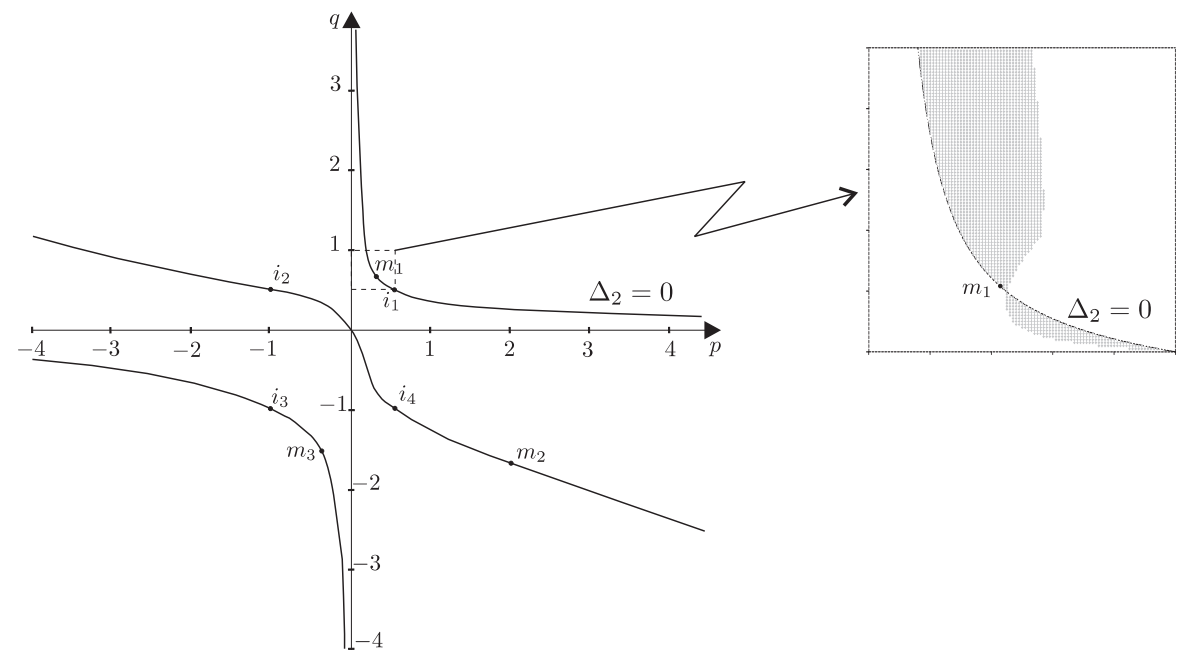

Figure 3: Location of the weak centers of system (2) for $\mu>1$ and, enlarged, a numerical study of a parameter subset not covered by Theorem A. The parameters in light grey have one critical period.

The above properties of $\Lambda_{1}, \Lambda_{2}$ and $\Lambda_{\infty}$ can be proved analytically. In Figure 3 we draw them qualitatively for $\mu>1$. This figure also contains a zoom of the square $\left[0, \frac{1}{2}\right] \times\left[\frac{1}{2}, 1\right]$ in which we make a numerical study $^{1}$ of the period function for $\mu=5.69$. For this value, $m_{1} \approx(0.21,0.61)$ and the light grey region corresponds to parameters for which the associated system has one critical period. (To be more precise, the ones below $\Delta_{2}=0$ correspond to a minimum and the ones above $\Delta_{2}=0$ to a maximum.) Note that the existence of this critical period near the curve $\Delta_{2}=0$ is established by $(a)$ in Theorem 3.1 because this curve consists precisely of weak centers of order 1 .

\subsection{Bifurcations at the outer boundary}

The bifurcations at the inner boundary for the family of centers in (2) are completely understood thanks to Theorem 3.1. In particular, the bifurcation curve is $\Delta_{2}=0$. The finding of its counterpart for the bifurcations at the outer boundary is a challenging problem. This is so already when the differential system is polynomial. However system (2) presents some features that makes this problem even more difficult.

\footnotetext{
${ }^{1}$ In fact we use system (9), which is conjugated to the original one and it has the center at the origin. We write it in polar coordinates as

$$
\left\{\begin{array}{l}
\dot{\theta}=\Theta(r, \theta), \\
\dot{r}=R(r, \theta) .
\end{array}\right.
$$

Then, if $r(\theta ; x)$ is the solution of $\frac{d r}{d \theta}=\frac{R(\theta, r)}{\Theta(r, \theta)}$ with initial condition $r(0 ; x)=x$, the period of the periodic orbit of system (9) passing through the point $(x, 0) \in \mathbb{R}^{2}$ is given by the definite integral

$$
T(x)=\int_{0}^{2 \pi} \frac{d \theta}{\Theta(r(\theta ; x), \theta)},
$$

where of course "everything" depends on $p, q$ and $\mu$. For $\mu=5.69$ we make a $100 \times 100$ grid on the square $\left[0, \frac{1}{2}\right] \times\left[\frac{1}{2}, 1\right]$ and, for each $(p, q)$ in this grid, we compute numerically $T$ in $m=400$ points equally spaced of its domain, say $x_{i}$ for $i=1,2, \ldots, m$. More concretely, we approximate the integral $T\left(x_{i}\right)$ by using the composite Simpson's rule with $n=500$ points in the interval $(0,2 \pi)$. To this end, for each $\theta_{k}=\frac{2 \pi k}{n}$ for $k=1,2, \ldots, n$, we compute using Newton's method $r\left(\theta_{k} ; x_{i}\right)=: r_{k}$ as a root of the equation $H\left(r_{k} \cos \theta_{k}, r_{k} \sin \theta_{k}\right)=H\left(x_{i}, 0\right)$, where $H(x, y)=F(x)+G(y)$ is the Hamiltonian function of system (9). Finally, once we have an approximation of $T\left(x_{i}\right)$ for $i=1,2, \ldots, m$, we use a five-point formula to approximate the derivative $T^{\prime}\left(x_{i}\right)$. The interested reader is referred to [21] for details about these numerical methods.
} 


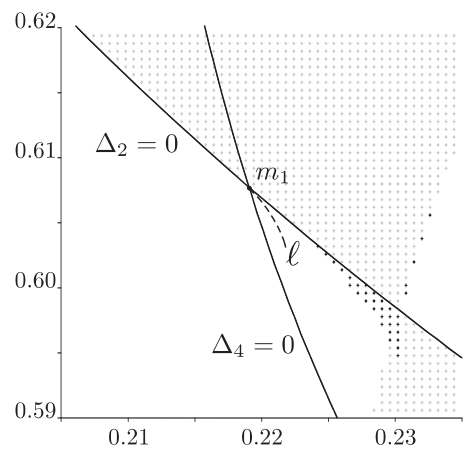

Figure 4: Numerical study near $m_{1}$ of the number of critical periods.

In general, even in case that the vector field is analytic at the polycycle, it turns out that the period function can not be extended there analytically. Then, instead of a Taylor series as in (10), the study leads to an asymptotic development of the period function in terms of the so-called Roussarie-Écalle compensator (see $[15,16,17])$. Of course this can be done in case that the vector field is defined at all the points of the polycycle, or at least such that we have some kind of extension there. (For instance, if $\mathcal{P}$ is unbounded then the polycycle has some vertices at infinity, where the vector field is not defined. This can be solved in case that the differential system is polynomial because then it can be extended meromorphically to the infinity by means of a suitable compactification.) In order to study the bifurcations at the outer boundary for system (2), the interesting cases for our purposes (i.e., the parameters not covered by Theorem A) present an additional difficulty apart from the ones we have just explained. Indeed, for these cases the outer boundary of the period annulus is a Jordan curve tangent to either $\{u=0\}$ or $\{v=0\}$, and the differential system is only continuous there (in fact there is not uniqueness of solutions). The tools developed in $[15,16,17]$ to study this type of bifurcation cannot be applied in such a degenerated setting.

\subsection{Bifurcations in the interior}

Figure 4 shows a zoom near $m_{1}$, a weak center of order 2 , where we made a numerical study of the period function for $\mu=5.69$. (Following the notation in the footnote, in this case we take a $50 \times 50$ grid with $m=1000$ and $n=500$.) As before, we color the parameters for which we detect one critical period in light grey. We also draw those parameters with two critical periods in black. We stress that a non-colored parameter does not mean the absence of critical periods. For instance, it may happen that there are two of them, but so close to each other that the change in the monotonicity of the period function can not be detected numerically because the discretization of its domain is not fine enough. This occurs precisely near a bifurcation of the period function in the interior of $\mathcal{P}$. The existence of a curve corresponding to this type of bifurcation landing at $m_{1}$ is the reason why we do not detect (numerically) parameters near $m_{1}$ having two critical periods as it is established by $(a)$ in Theorem 3.1.

One can verify, see Figure 4 , that $\Delta_{2}=0$ and $\Delta_{4}=0$ intersect transversally at $m_{1}$. Taking this fact into account, it can be proved that there exists a germ of curve $\ell$ at $m_{1}$ tangent to $\Delta_{2}=0$ and inside $\left\{\Delta_{2}<0\right\} \cap\left\{\Delta_{4}>0\right\}$ which corresponds to bifurcations in the interior of $\mathcal{P}$. (The proof follows by applying the Weierstrass Preparation Theorem exactly the same way as in Theorem 4.3 of [16].) According to the simplified model that we explain at the beginning of the Section, this curve should join $\Delta_{2}=0$ with the curve corresponding to bifurcations at the outer boundary. Note finally that since $\Delta_{2}=0$ and $\Delta_{4}=0$ also intersect transversally at $m_{3}$, we can assert the existence of another germ of this type of curve near $m_{3}$. 


\section{References}

[1] X. Chen and W. Zhang, Decomposition of algebraic sets and applications to weak centers of cubic systems, J. Comput. Appl. Math. 232 (2009), 565-581.

[2] C. Chicone, The monotonicity of the period function for planar Hamiltonian vector fields, J. Differential Equations 69 (1987), 310-321.

[3] C. Chicone and M. Jacobs, Bifurcation of critical periods for plane vector fields, Trans. Amer. Math. Soc. 312 (1989), 433-4

[4] C.J. Christopher and C.J. Devlin, Isochronous centres in planar polynomial systems, SIAM Jour. Math. Anal. 28 (1997), 162-177.

[5] A. Cima, A. Gasull and F. Mañosas, Period function for a class of Hamiltonian systems, J. Differential Equations 168 (2000), 180ñ-199.

[6] A. Cima, F. Mañosas and J. Villadelprat, Isochronicity for several classes of Hamiltonian systems, J. Differential Equations 157 (1999), 373-413.

[7] A. Dancsó, H. Farkas, M. Farkas and G. Szabó, Investigations into a class of generalized two-dimensional Lotka-Volterra schemes, Acta Appl. Math. 23 (1991), 103-127.

[8] H. Eyring and D. Henderson, "Theoretical chemistry, volume 4, periodicities in chemistry and biology", Academic Press, New York, 1978.

[9] H. Farkas and Z. Noszticzius, Generalized Lotka-Volterra schemes and the construction of twodimensional explodator cores and their Lyapunov functions via "critical" Hopf bifurcations, J. Chem. Soc. Faraday Trans. II 81 (1985), 1487-1505.

[10] R. Field and M. Burger, "Oscillations and traveling waves in chemical systems", Wiley, New York, 1985.

[11] E. Freire, A. Gasull and A. Guillamon, First derivative of the period function with applications, J. Differential Equations 204 (2004), 139-162.

[12] A. Gasull and Y. Zhao, Bifurcation of critical periods from the rigid quadratic isochronous vector field, Bull. Sci. Math. 132 (2008),292-312.

[13] M. Grau and J. Villadelprat, Bifurcation of critical periods from Pleshkan's isochrones, to appear in J. London Math. Soc. (2009).

[14] P. Mardešić, L. Moser-Jauslin and C. Rousseau, Darboux linearization and isochronous centers with a rational first integral, J. Differential Equations 134 (1997), 216-268.

[15] P. Mardešić,, D. Marín and J. Villadelprat, On the time function of the Dulac map for families of meromorphic vector fields, Nonlinearity 16 (2003), 855-881.

[16] P. Mardešić,, D. Marín and J. Villadelprat, The period function of reversible quadratic centers, J. Differential Equations 224 (2006), 120-171.

[17] P. Mardešić,, D. Marín and J. Villadelprat, Unfolding of resonant saddles and the Dulac time, Discrete Contin. Dyn. Syst. 21 (2008), 1221-1244.

[18] F. Rothe, The periods of the Volterra-Lokta system, J. Reine Angew. Math. 355 (1985), 129-138.

[19] C. Rousseau and B. Toni, Local bifurcation of critical periods in vector fields with homogeneous nonlinearities of the third degree, Canad. Math. Bull. 36 (1993), 473-484. 
[20] R. Schaaf, Global behaviour of solution branches for some Neumann problems depending on one or several parameters, J. Reine Angew. Math. 346 (1984), 1-31.

[21] J. Stoer and R. Bulirsch, "Introduction to numerical analysis", Texts in Applied Mathematics, 12, Springer-Verlag, New York, 2002.

[22] J. Villadelprat, On the reversible quadratic centers with monotonic period function, Proc. Amer. Math. Soc. 135 (2007), 2555-2565.

[23] J. Villadelprat, The period function of the generalized Lotka-Volterra centers, J. Math. Anal. Appl. 341 (2008), 834-854.

[24] J. Waldvogel, The period in the Lotka-Volterra system is monotonic, J. Math. Anal. Appl. 114 (1986), $178-184$.

[25] Z. Wang, X. Chen and W. Zhang, Local bifurcations of critical periods in a generalized 2D LV system, Appl. Math. Comput. 214 (2009), 17-25.

[26] A. Winfree, "The geometry of biological time", Interdisciplinary Applied Mathematics, 12, SpringerVerlag, New York, 2001.

[27] W. Zhang, X. Hou and Z. Zeng, Weak centers and bifurcation of critical periods in reversible cubic systems, Comput. Math. Appl. 40 (2000), 771-782.

[28] Y. Zhao, On the monotonicity of the period function of a quadratic system, Discrete Contin. Dyn. Syst. 13 (2005), 795-810.

[29] H. Zoladek, Quadratic systems with center and their perturbations, J. Differential Equations 109 (1994), $223-273$.

[30] L. Zou, X. Chen and W. Zhang, Local bifurcations of critical periods for cubic Liénard equations with cubic damping, J. Comput. Appl. Math. 222 (2008), 404-410. 